\title{
Evaluación comparativa del ciclo de vida de cuatro soluciones constructivas diferentes para la rehabilitación de pisos de viguetas de madera con valor patrimonial
}

\section{A comparative life cycle assessment of four different constructive solutions for the rehabilitation of planked timber floors with heritage value}

\author{
C. M. Calama-González ${ }^{(*)}$, C. Cañas Palop ${ }^{(* *)}$
}

\section{RESUMEN}

El sector de la construcción en España es un gran consumidor de recursos naturales y energéticos, siendo responsable del 50\% de los residuos generados. La apuesta de las normativas actuales por un desarrollo sostenible, conlleva a que el Análisis de Ciclo de Vida (ACV) sea una herramienta fundamental para aproximarse a criterios medioambientales y valorar las intervenciones previamente al proceso constructivo. Este trabajo plantea la evaluación de los impactos medioambientales de cuatro soluciones de rehabilitación de forjados con entrevigado abovedado en edificios protegidos y de interés patrimonial de los S. XVII-XIX, en Sevilla. Tres de las soluciones de rehabilitación seleccionadas son de empleo común en este tipo de intervenciones y la cuarta utiliza una pieza prefabricada de entrevigado elaborada con un mortero cerámico reciclado. Este trabajo demuestra un mejor comportamiento de la última solución, al tener menores impactos ambientales y ser compatible con criterios patrimoniales.

Palabras clave: Análisis del ciclo de vida, residuos de cerámica, rehabilitación, forjado de madera, patrimonio.

\section{ABSTRACT}

The Spanish construction sector is a huge consumer of natural and energy resources, being responsible for 50\%f of the total generated waste. Due to current regulations for sustainable development, Life Cycle Assessment (LCA) is a fundamental tool to approach environmental criteria and assess interventions prior to construction. This paper evaluates the environmental impacts of four solutions for rehabilitating timber floors, with pieces between beams in curved form, in heritage buildings from the 1700 s to 1900 s, in Seville. Three of the proposed rehabilitation solutions are widely used in these type of interventions and the fourth uses prefabricated pieces made from a recycled ceramic mortar, as elements between beams. Results show a better environmental performance of the recycled solution, also being compatible with patrimonial criteria.

Keywords: Life Cycle Assessment, Ceramic waste, Rehabilitation, Planked timber floor, heritage.

(*) Arquitecto. PDI. IUACC, E.T.S. Arquitectura, Universidad de Sevilla, Spain.

$\left({ }^{* *}\right)$ Doctor Arquitecto. PDI. Dpto. Construcciones Arquitectónicas, Universidad de Sevilla, Spain.

Persona de contacto/Corresponding author: ccalama@us.es (C. Calama).

ORCID: https://orcid.org/oooo-0oo2-6511-2885 (C. M. Calama-González); https://orcid.org/oooo-0oo2-5171-4471

(C. Cañas Palop).

Cómo citar este artículo/Citation: Calama-González, C. M.; Cañas Palop, C. (2019). Evaluación comparativa del ciclo de vida de cuatro soluciones constructivas diferentes para la rehabilitación de pisos de viguetas de madera con valor patrimonial. Informes de la Construcción, 71(556): e316. https://doi.org/10.3989/ic.66752.

Copyright: ( ) 2019 CSIC. Este es un artículo de acceso abierto distribuido bajo los términos de la licencia de uso y distribución Creative Commons Reconocimiento 4.0 Internacional (CC BY 4.0). 


\section{INTRODUCCIÓN}

Actualmente, el sector de la construcción española consume en torno al $40 \%$ de las materias primas extraídas (1) y contribuye en la emisión de hasta el $36 \%$ de $\mathrm{CO}_{2}$ total (2), siendo responsable de cerca del $40 \%$ de la energía final consumida (3) y representando aproximadamente un 50\% de los residuos generados en el país (4). Además, el tercer material más empleado en la edificación es el cerámico (5), generándose en Europa unos 450 millones de toneladas anuales de residuos cerámicos, de entre los cuales 3 millones proceden de desechos del proceso de fabricación.

Entre las mejoras de las edificaciones basadas en criterios de sostenibilidad, se integra la reutilización de materiales para minimizar los consumos energéticos de la extracción de recursos naturales y de los procesos de fabricación y tratamiento. Pese a estar avalada por numerosas legislaciones (6) y existir una notable cantidad de publicaciones científicas relativas al reciclaje de materiales cerámicos para la creación de morteros y hormigones reciclados (7) (8), la puesta en valor de los residuos en España es todavía escasa: con una cifra de recuperación del $27,1 \%$, casi un $15 \%$ menos de la media europea (9).

En este contexto, el Análisis de Ciclo de Vida (ACV) adquiere gran importancia para la evaluación de los impactos medioambientales (10), según la normativa de aplicación (11), y para la toma de decisiones, ofreciendo información para determinar los impactos preferibles. Esto, a la vez, potencia la competitividad en el sector comercial, apostando por soluciones cada vez más eco-eficientes (12).

Durante los últimos años, se han estudiado ampliamente los impactos ambientales de los productos cerámicos, generalmente mediante un análisis desde la cuna a la tumba. Algunas publicaciones se centran en materiales concretos, como baldosas (13), ladrillos (14) o tejas (15). En otras ocasiones, se realiza un estudio comparativo de materiales, por ejemplo evaluando $1 \mathrm{~kg}$ de productos cerámicos, de hormigón o de fibrocemento, $1 \mathrm{~m}^{2}$ de tejas cerámicas o de hormigón (16) o la huella ecológica de un ladrillo, una teja y una baldosa cerámica (17).

No obstante, es más frecuente analizar productos constructivos, principalmente soluciones de fachada, como paneles cerámicos frente a otros de vidrio, mármol, aluminio u hormigón (18). Existe un número más escaso de trabajos donde se analizan soluciones de cubierta (19) y particiones (20), incorporando de una u otra forma la cerámica. En cuanto a forjados, no se ha encontrado ningún ACV con este material. Por último, las publicaciones que analizan edificaciones en las que se emplean materiales cerámicos, bien como elementos estructurales (21), bien como revestimientos (22), son bastante reducidas.

En las políticas actuales de rehabilitación de edificaciones en cascos históricos, cobra pues notable interés el ACV enfocado al reciclaje de materiales. Sin embargo, la gran mayoría de los estudios están referidos a obra de nueva planta o materiales no tradicionales. Es decir, apenas se ha estudiado la rehabilitación de edificios existentes a través de la técnica del ACV como estrategia de sostenibilidad ambiental. De hecho, es frecuente que la evaluación de los impactos ambientales se refieran principalmente a rehabilitaciones energéticas de los edificios (fundamentalmente la envolvente vertical, al ser normalmente el elemento con mayor predominio en el conjunto), estando relegadas a un segundo plano otro tipo de intervenciones, como las vinculadas a elementos estructurales (23). Por consiguiente, la presente investigación aporta una visión innovadora en el campo de estudio del ciclo de vida, desde la perspectiva de la rehabilitación de edificios con valor patrimonial, al incluir el ACV como criterio medioambiental para la toma de decisiones en el proceso rehabilitador de elementos estructurales.

\section{MATERIALES Y MÉTODOS}

\subsection{Definición de objetivos y alcance}

El objetivo de esta investigación es la evaluación de las cargas medioambientales asociadas a una solución constructiva, que se emplea como elemento estructural horizontal (forjado intermedio), en la que se implementa una pieza de entrevigado. Esta pieza se ha elaborado a partir de un mortero con residuos cerámicos, siendo previamente analizada y ensayada en laboratorio (24). Su aplicación final está orientada a la rehabilitación de forjados unidireccionales de entrevigado abovedado o de revoltón con viguería de madera, muy frecuentes en la arquitectura doméstica del sur de España y, en especial, de Sevilla de los S. XVII-XIX (25). Además, en este ACV se compara la citada solución con otras tres posibles opciones de rehabilitación que ofrece el sector comercial actual para este tipo de intervenciones patrimoniales, de gran relevancia en la ciudad.

\subsubsection{Límites del sistema}

El alcance del estudio se centra en un primer análisis de factores medioambientales, que permitan obtener información válida para la toma de decisiones en la rehabilitación arquitectónica de forjados con entrevigado abovedado. Por ello, como unidad funcional se ha evaluado el impacto considerando las "toneladas de producto final por cada $\mathrm{m}^{2}$ de forjado construido, cumpliendo las exigencias y estándares mínimos de la normativa de aplicación". También hay que tener presente que el grado de conservación de la estructura original que permiten las diferentes soluciones analizadas, puede repercutir en el cómputo global del ACV.

Asimismo, se ha de dejar constancia que debido a la extensión del trabajo, en el presente artículo únicamente se plantea una aproximación al estudio medioambiental de las soluciones constructivas, por lo que se presentan los resultados de la evaluación de los impactos medioambientales referidos a la fase de pre-ocupación. Es decir, desde la extracción de materias primas (A1) a la fabricación del material o producto (A3) y el transporte a fábrica y a la obra (A2 y A4, respectivamente). En esta elección se ha tenido en cuenta que a estas fases se les asocia los mayores impactos medioambientales y, por tanto, resultan las más significativas para el estudio, aspecto en el que prácticamente la totalidad de las publicaciones en la materia coinciden (26). Los límites del sistema que han sido considerados se muestran en la Figura 1.

\subsubsection{Metodología de evaluación del impacto.}

La metodología empleada en este ACV se corresponde con la establecida en la ISO 14040:2006 y tiene en cuenta los requisitos y aspectos indicados en la ISO 14044:2006 (Ver 


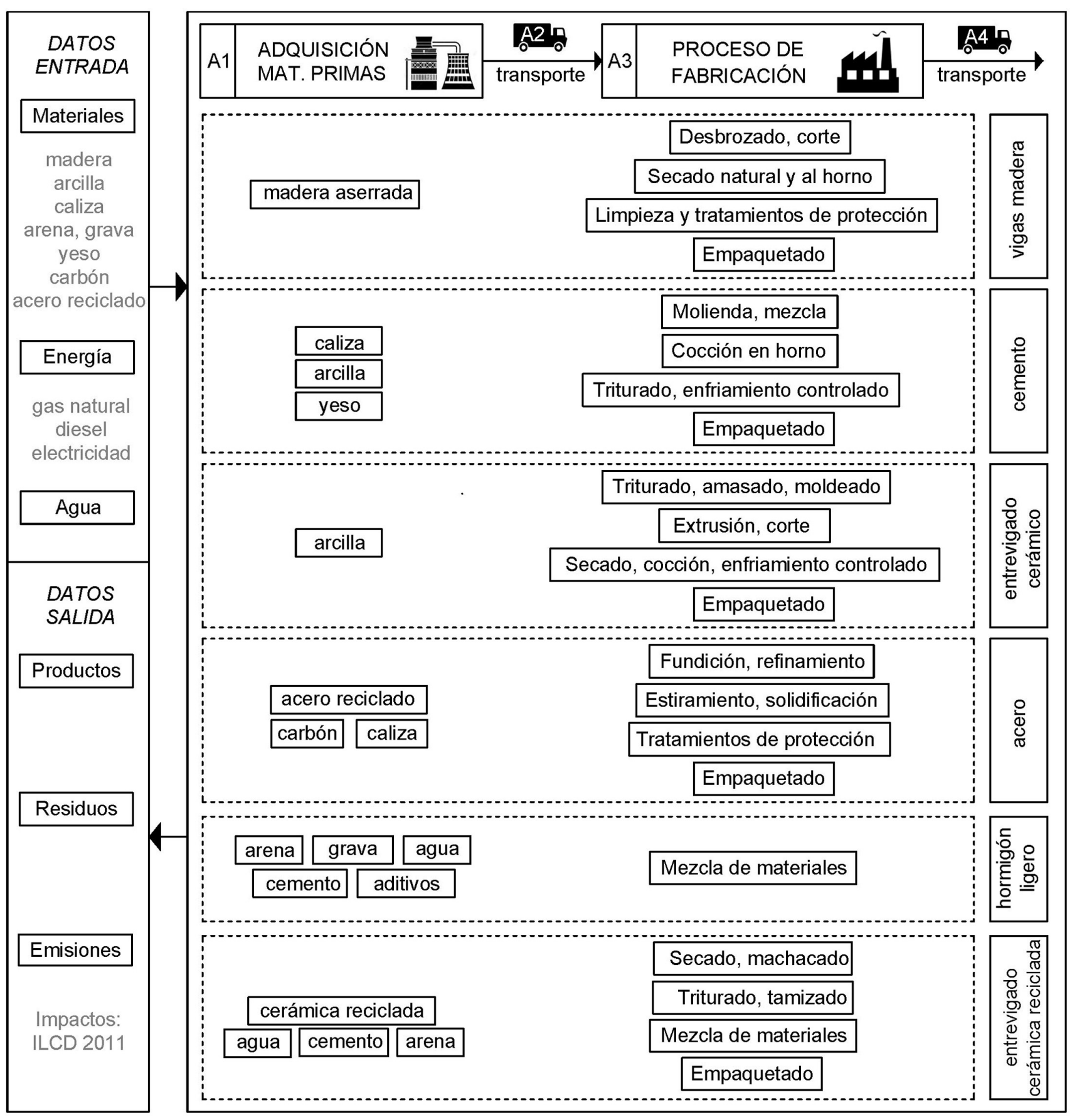

Figura 1. Límites del sistema, entradas y salidas consideradas en el ACV. Fuente: autores.

Referencia 11). Las categorías de impacto analizadas están incluidas en el ILCD 2011, sistema internacional de referencia europeo sobre el ciclo de vida. En el procedimiento de cálculo del ACV, se ha empleado el programa informático de código abierto openLCA versión 1.6.1 (Mozilla Public Licence 2.0, by GreenDelta) (27)

\section{ANÁLISIS DEL INVENTARIO}

\subsection{Soluciones constructivas consideradas}

La Figura 2 muestra las cuatro soluciones de rehabilitación analizadas, cuya elección se ha basado en criterios comúnmente empleados en el ámbito local para la rehabilitación de forjados de revoltón, en los que se utilicen piezas cerámicas de entrevigado.
En primer lugar, se han considerado dos hipótesis de sustitución total del forjado, siendo posible la incorporación de piezas de entrevigado de tipo cerámico de dimensiones estandarizadas (entre $50-80 \mathrm{~cm}$ ), superiores a las normalmente empleadas en obras de rehabilitación (inferiores a los $50 \mathrm{~cm}$ ).

En la solución 1a se utiliza una bovedilla cerámica convencional aligerante, mientras que en la $1 \mathrm{~b}$ se ha escogido un entrevigado cerámico macizo curvo, tipo mallorquín. Las dos hipótesis restantes permiten una sustitución parcial del forjado, conservando las vigas de madera originales y la separación interejes, es decir, teniendo en cuenta criterios patrimoniales, constructivos, morfológicos y/o tipológicos que pueden venir impuestos por normativas urbanísticas y catálogos de protección. En la solución 1c se propone la ejecución in situ de bóvedas tabicadas de ladrillo, empleando técnicas constructivas tradicionales. Por 

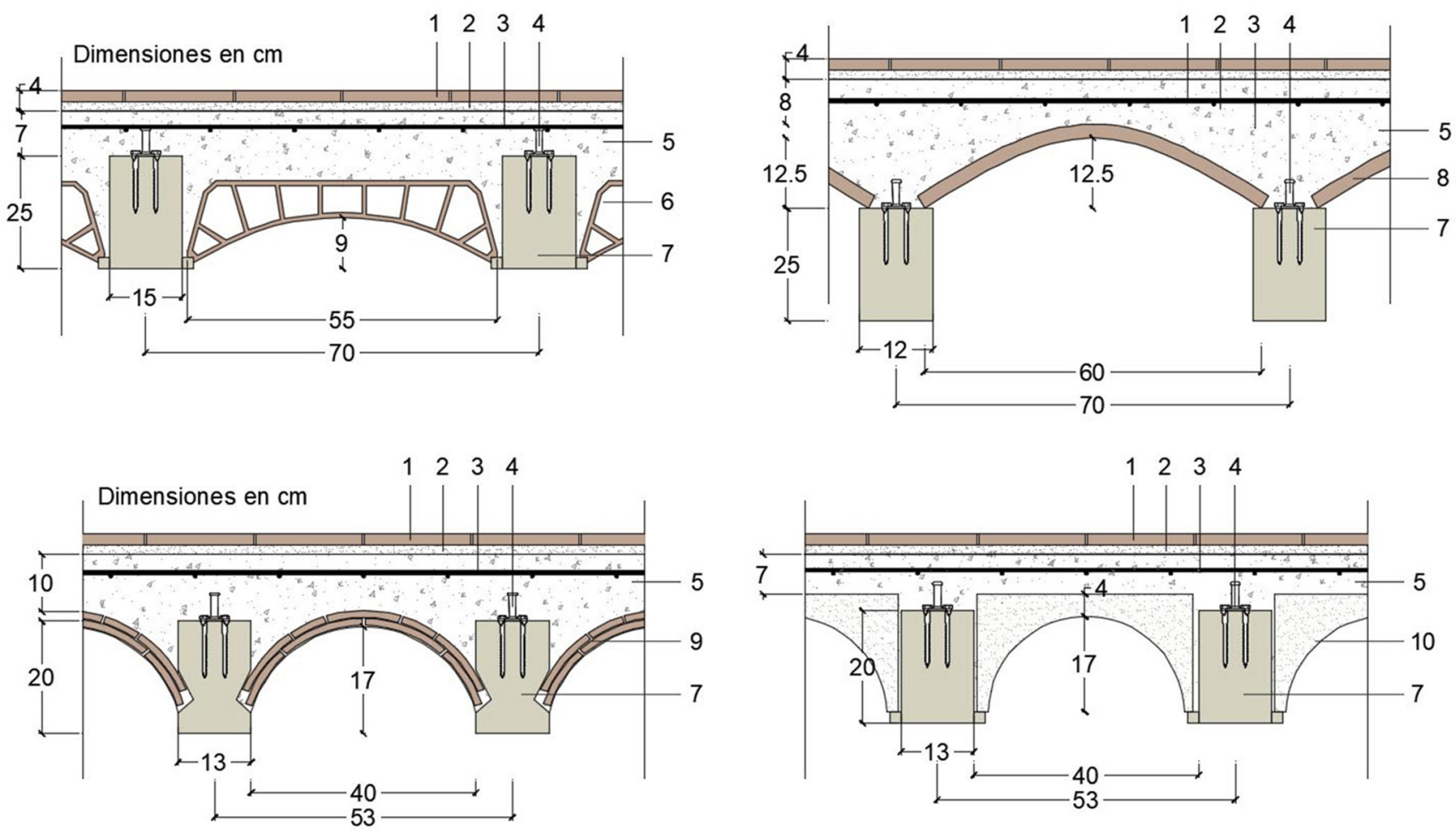

1. Solería de balsosas cerámicas, $2 \mathrm{~cm}$

2. Mortero de cemento, $2 \mathrm{~cm}$

3. Refuerzo de acero corrugado $20 \times 20$ A Ø6-6 B400S

4. Conectores de acero galvanizado

5. Capa de compresión: relleno de hormigón ligero, HLE-25/B/10/lla, $1.200 \mathrm{~kg} / \mathrm{m}^{3}$
6. Entrevigado cerámico: piezas aligeradas, $1.800 \mathrm{~kg} / \mathrm{m}^{3}$

7. Vigas de madera, $370 \mathrm{~kg} / \mathrm{m}^{3}$

8. Entrevigado cerámico: piezas macizas, $1.800 \mathrm{~kg} / \mathrm{m}^{3}$

9. Entrevigado cerámico: baldosas cerámicas, $1.800 \mathrm{~kg} / \mathrm{m}^{3}$

10. Entrevigado prefabricado con un $30 \%$ de residuos cerámicos

Figura 2. Hipótesis de las soluciones constructivas consideradas para la rehabilitación de forjados con entrevigado abovedado, evaluadas mediante el ACV: (a) Bovedilla cerámica aligerante; (b) Bovedilla cerámica maciza; (c) Bovedilla con losas cerámicas; (d): Bovedilla cerámica reciclada. Fuente: autores.

último, en la hipótesis 1d se implementa una pieza de entrevigado cerámico reciclado diseñada y analizada por el grupo de investigación TEP-205 Análisis y Evaluación de Sistemas Constructivos y Estructurales en la Arquitectura, de la Universidad de Sevilla, que ha sido recientemente patentada (28).

Es importante indicar que la normativa actual en materia de urbanismo y las fichas de protección del conjunto histórico de Sevilla (29), promueven la conservación y rehabilitación del parque edificado, especialmente numeroso en el centro histórico de la ciudad. En la arquitectura doméstica, aunque puede haber variaciones según el grado de protección, es muy frecuente que las exigencias de rehabilitación se refieran a la conservación de la fachada, de la primera crujía y de la morfología del forjado. En este último caso, fundamentalmente se trata de aspectos estéticos, separación a intereje y, en la medida de lo posible, la conservación de elementos originales. Sin embargo, los criterios patrimoniales actuales se basan fundamentalmente en principios formales, sin tener en cuenta medidas de sostenibilidad vinculadas al consumo energético de recursos o requisitos medioambientales.

En este sentido, de las cuatro soluciones analizadas, las tres primeras se corresponden con las empleadas con mayor frecuencia en la rehabilitación de edificios patrimoniales con forjados de viguería de madera y entrevigado abovedado de la arquitectura de Sevilla de los S. XVII-XIX.
Las soluciones 1c y 1d, permiten mantener la tipología del forjado de acuerdo con las normativas urbanísticas y catálogos de protección anteriormente citados. La razón es que permiten optar por una sustitución parcial del forjado y conservar la separación interejes, así como la viguería de madera original u otros elementos vistos que se encuentren en buen estado.

En la Tabla 1 se presenta el cálculo de los diferentes materiales empleados por m2 de forjado en cada una de las cuatro hipótesis de rehabilitación. Para que la comparativa entre las soluciones sea adecuada y, partiendo del análisis de criterios empleados en obras de rehabilitación, en todas las hipótesis se utilizarán materiales con las mismas características técnicas. Además, las cuatro soluciones han sido evaluadas estructuralmente a: Estado Límite Último (flexión, cortante y rasante), Estado Límite de Servicio (flecha) y frente al comportamiento al fuego (R-6o). Las cuatro soluciones se han dimensionado para cumplir con los requisitos exigidos por la normativa española vigente (30).

En concreto, se ha considerado un refuerzo estructural mediante una losa superior de hormigón armado, es decir, un forjado colaborante, que permita conservar la estética y morfología de la cara inferior del forjado a rehabilitar, y cuyo espesor se ha calculado específicamente para cada una de las hipótesis según sus propias cargas y características. 


\subsection{Inventario del ciclo de vida}

Los datos cuantitativos empleados en el cálculo del ACV (Tablas 2 y 3) han sido obtenidos de la Base de Datos del Insti- tuto de Tecnología de la Construcción de Cataluña (31), que incorpora una Base de Información Ambiental de productos y sistemas de la construcción (32), y de la base del proyecto europeo inData de OpenDAP (33).

Tabla 1. Masa de los materiales empleados en la rehabilitación de $1 \mathrm{~m} 2$ de forjado para las cuatro soluciones constructivas estudiadas.

\begin{tabular}{|c|c|c|c|c|c|}
\hline Solución de rehabilitación & \multicolumn{2}{|c|}{ Material (por m²) } & Volumen $\left(\mathrm{m}^{3}\right)$ & Densidad $\left(\mathrm{kg} / \mathrm{m}^{3}\right)$ & $\operatorname{Masa}\left(t / \mathbf{m}^{2}\right)$ \\
\hline \multirow{5}{*}{$\begin{array}{c}1 \mathrm{a} \\
\left(3.00 \mathrm{kN} / \mathrm{m}^{2}\right)\end{array}$} & \multicolumn{2}{|c|}{ Madera aserrada (reemplazada) } & 0.077 & 370 & 0.03 \\
\hline & \multicolumn{2}{|c|}{ Pieza de entrevigado cerámica } & 0.019 & 1800 & 0.03 \\
\hline & \multicolumn{2}{|c|}{ Hormigón ligero HLE-25/P/20/IIa } & 0.121 & 1200 & 0.15 \\
\hline & \multicolumn{2}{|c|}{ Refuerzo de acero corrugado B40oS } & 0.048 & 7850 & 0.38 \\
\hline & \multicolumn{2}{|c|}{ Conectores de acero galvanizado } & 0.032 & 7850 & 0.25 \\
\hline \multirow{5}{*}{$\begin{array}{c}1 \mathrm{~b} \\
\left(3.30 \mathrm{kN} / \mathrm{m}^{2}\right)\end{array}$} & \multicolumn{2}{|c|}{ Madera aserrada (reemplazada) } & 0.060 & 370 & 0.02 \\
\hline & \multicolumn{2}{|c|}{ Pieza de entrevigado cerámica } & 0.023 & 1800 & 0.04 \\
\hline & \multicolumn{2}{|c|}{ Hormigón ligero HLE-25/P/20/IIa } & 0.100 & 1200 & 0.12 \\
\hline & \multicolumn{2}{|c|}{ Refuerzo de acero corrugado B40oS } & 0.048 & 7850 & 0.38 \\
\hline & \multicolumn{2}{|c|}{ Conectores de acero galvanizado } & 0.031 & 7850 & 0.24 \\
\hline \multirow{5}{*}{$\begin{array}{c}1 \mathrm{c} \\
\left(3.20 \mathrm{kN} / \mathrm{m}^{2}\right)\end{array}$} & \multicolumn{2}{|c|}{ Madera aserrada (conservada) } & 0.048 & 370 & 0.02 \\
\hline & \multicolumn{2}{|c|}{ Pieza de entrevigado cerámica } & 0.023 & 1800 & 0.04 \\
\hline & \multicolumn{2}{|c|}{ Hormigón ligero HLE-25/P/20/IIa } & 0.152 & 1200 & 0.18 \\
\hline & \multicolumn{2}{|c|}{ Refuerzo de acero corrugado B40oS } & 0.048 & 7850 & 0.38 \\
\hline & \multicolumn{2}{|c|}{ Conectores de acero galvanizado } & 0.025 & 7850 & 0.20 \\
\hline \multirow{8}{*}{$\begin{array}{c}1 \mathrm{~d} \\
\left(2.75 \mathrm{kN} / \mathrm{m}^{2}\right)\end{array}$} & \multicolumn{2}{|c|}{ Madera aserrada (conservada) } & 0.077 & 370 & 0.03 \\
\hline & \multirow{4}{*}{$\begin{array}{c}\text { Pieza de } \\
\text { entrevigado } \\
\text { cerámico reciclado }\end{array}$} & Cerámica & 0.014 & 1500 & 0.02 \\
\hline & & Arena & 0.003 & 1400 & 0.04 \\
\hline & & Cemento & 0.008 & 1600 & 0.01 \\
\hline & & Agua & 0.008 & 1000 & 0.01 \\
\hline & \multicolumn{2}{|c|}{ Hormigón ligero HLE-25/P/20/IIa } & 0.031 & 1200 & 0.04 \\
\hline & \multicolumn{2}{|c|}{ Refuerzo de acero corrugado B40oS } & 0.048 & 7850 & 0.38 \\
\hline & \multicolumn{2}{|c|}{ Conectores de acero galvanizado } & 0.032 & 7850 & 0.25 \\
\hline
\end{tabular}

Tabla 2. Datos de entrada empleados en la etapa A1 del ACV en función de cada material.

\begin{tabular}{|c|c|c|c|}
\hline Material & A1 Extracción de Materias Primas & Cantidad & Unidad \\
\hline \multirow{3}{*}{ Madera aserrada } & Materia prima: madera aserrada & 1.20 & $\mathrm{t} / \mathrm{t}$ producto final \\
\hline & Combustible: diesel & 10.63 & l/t producto final \\
\hline & Energía consumida & 435.25 & $\mathrm{MJ} / \mathrm{t}$ producto final \\
\hline \multirow{5}{*}{$\begin{array}{c}\text { Cemento } \\
\text { L II/A-L 42,5 R }\end{array}$} & Materia prima: caliza & 1.60 & \multirow{3}{*}{$\mathrm{t} / \mathrm{t}$ producto final } \\
\hline & Materia prima secundaria: arcilla & 0.40 & \\
\hline & Aditivo y yeso & 0.06 & \\
\hline & Combustible: diesel & 0.81 & $\mathrm{l} / \mathrm{t}$ producto final \\
\hline & Energía consumida & 7.10 & $\mathrm{MJ} / \mathrm{t}$ producto final \\
\hline \multirow{3}{*}{ Cerámica } & Materia prima: arcilla & 1.25 & t/t producto final \\
\hline & Combustible: diesel & 0.49 & l/t producto final \\
\hline & Energía consumida & 20.06 & $\mathrm{MJ} / \mathrm{t}$ producto final \\
\hline \multirow{3}{*}{ Arena de río lavada } & Materia prima: arena & 1.00 & $\mathrm{t} / \mathrm{t}$ producto final \\
\hline & Combustible: diesel & 3.66 & $\mathrm{l} / \mathrm{t}$ producto final \\
\hline & Energía consumida & 150.00 & $\mathrm{kWh} / \mathrm{t}$ producto final \\
\hline \multirow{3}{*}{ Grava } & Materia prima: grava & 1.00 & $\mathrm{t} / \mathrm{t}$ producto final \\
\hline & Combustible: diesel & 3.66 & l/t producto final \\
\hline & Energía consumida & 150.00 & $\mathrm{kWh} / \mathrm{t}$ producto final \\
\hline \multirow{4}{*}{ Refuerzo de acero corrugado B40oS } & Materia prima: acero reciclado & 0.88 & \multirow{3}{*}{$\mathrm{t} / \mathrm{t}$ producto final } \\
\hline & Materia prima secundaria: carbón & 0.15 & \\
\hline & Materia prima secundaria: caliza & 0.04 & \\
\hline & Energía consumida & 4.50 & $\mathrm{MJ} / \mathrm{t}$ producto final \\
\hline \multirow{4}{*}{ Conectores de acero galvanizado } & Materia prima: acero reciclado & 0.88 & \multirow{3}{*}{$\mathrm{t} / \mathrm{t}$ producto final } \\
\hline & Materia prima secundaria: carbón & 0.15 & \\
\hline & Materia prima secundaria: caliza & 0.04 & \\
\hline & Energía consumida & 4.50 & $\mathrm{MJ} / \mathrm{t}$ producto final \\
\hline \multirow{3}{*}{ Hormigón ligero HLE-25/P/20/IIa } & Materia prima: cemento & 0.15 & \multirow{3}{*}{$\mathrm{t} / \mathrm{t}$ producto final } \\
\hline & Materia prima: arena & 0.30 & \\
\hline & Materia prima: grava & 0.60 & \\
\hline Cerámica reciclada & Materia prima: cerámica & $0.00^{*}$ & $\mathrm{t} / \mathrm{t}$ producto final \\
\hline
\end{tabular}

* Los residuos cerámicos que se pierden en el proceso de fabricación se corresponde con un 25\% de lo fabricado. El empleo de cerámica reciclada, implica que no existen consumos energéticos durante el proceso de extracción de las materias primas. 
Tabla 3. Datos de entrada empleados en la etapa A3 del ACV en función de cada material.

\begin{tabular}{|c|c|c|c|}
\hline Material & A3 Fabricación del Producto & Cantidad & Unidad \\
\hline \multirow{3}{*}{ Madera aserrada } & Energía consumida & 971.22 & $\mathrm{MJ} / \mathrm{t}$ producto final \\
\hline & Agua consumida & 2000.00 & \multirow{2}{*}{$1 / t$ producto final } \\
\hline & Combustible: diesel & 23.72 & \\
\hline \multirow{3}{*}{$\begin{array}{c}\text { Cemento } \\
\text { BL II/A-L 42,5 R }\end{array}$} & Energía consumida & 4270.00 & $\mathrm{MJ} / \mathrm{t}$ producto final \\
\hline & Agua consumida & 30000.00 & \multirow{2}{*}{ l/t producto final } \\
\hline & Combustible: diesel & 104.30 & \\
\hline \multirow{3}{*}{ Cerámica } & Energía consumida & 3453.03 & $\mathrm{MJ} / \mathrm{t}$ producto final \\
\hline & Agua consumida & 200.00 & l/t producto final \\
\hline & Combustible: gas natural & 82.49 & $\mathrm{~m} 3 / \mathrm{t}$ producto final \\
\hline \multirow{3}{*}{ Arena de río lavada } & Energía consumida & 20.00 & $\mathrm{MJ} / \mathrm{t}$ producto final \\
\hline & Agua consumida & 2500.00 & \multirow{2}{*}{ l/t producto final } \\
\hline & Combustible: diesel & 0.49 & \\
\hline \multirow{3}{*}{ Grava } & Energía consumida & 20.00 & $\mathrm{MJ} / \mathrm{t}$ producto final \\
\hline & Agua consumida & 1600.00 & \multirow{2}{*}{ l/t producto final } \\
\hline & Combustible: diesel & 0.49 & \\
\hline \multirow{2}{*}{ Refuerzo de acero corrugado } & Energía consumida & 29200.00 & $\mathrm{MJ} / \mathrm{t}$ producto final \\
\hline & Agua consumida & 95000.00 & $\mathrm{l} / \mathrm{kg}$ producto final \\
\hline \multirow{6}{*}{ Conectores de acero galvanizado } & Energía consumida & 29400.00 & $\mathrm{MJ} / \mathrm{t}$ producto final \\
\hline & Agua consumida & 95010.00 & $\mathrm{l} / \mathrm{kg}$ producto final \\
\hline & Materia prima: acero reciclado & 0.88 & \multirow{3}{*}{ t/t producto final } \\
\hline & Materia prima secundaria: carbón & 0.15 & \\
\hline & Materia prima secundaria: caliza & 0.04 & \\
\hline & Energía consumida & 4.50 & $\mathrm{MJ} / \mathrm{t}$ producto final \\
\hline \multirow{6}{*}{$\begin{array}{l}\text { Hormigón ligero } \\
\text { HLE-25/P/20/IIa }\end{array}$} & Energía consumida & 1130.00 & $\mathrm{MJ} / \mathrm{t}$ producto final \\
\hline & Agua consumida & 75.00 & \multirow{2}{*}{ l/t producto final } \\
\hline & Combustible: diesel & 29.20 & \\
\hline & Materia prima: cemento & 0.15 & \multirow{3}{*}{$\mathrm{t} / \mathrm{t}$ producto final } \\
\hline & Materia prima: arena & 0.30 & \\
\hline & Materia prima: grava & 0.60 & \\
\hline \multirow{3}{*}{ Cerámica reciclada } & Energía consumida & 381.29 & $\mathrm{MJ} / \mathrm{t}$ producto final \\
\hline & Combustible: gas natural & 0.95 & $\mathrm{~m} 3 / \mathrm{t}$ producto final \\
\hline & Materia prima: residuo cerámico & 0.00 & t/t producto final \\
\hline
\end{tabular}

Tabla 4. Escenarios de transporte (A2 y A4) considerados en las cuatro soluciones.

\begin{tabular}{|c|c|c|c|c|}
\hline Material & \multicolumn{2}{|c|}{$\begin{array}{c}\text { A2 Distancia de Transporte } \\
\text { (A Fábrica) }\end{array}$} & \multicolumn{2}{|c|}{$\begin{array}{c}\text { A4 Distancia de Transporte } \\
\text { (A Obra) }\end{array}$} \\
\hline Madera aserrada & $2.00 \mathrm{~km}$ & Camion. $75+6028 \mathrm{MI} / \mathrm{h}$ & $830.00 \mathrm{~km}$ & \multirow{7}{*}{ Camión: 7.5 t, $62.38 \mathrm{MJ} / \mathrm{h}$} \\
\hline Cemento BL II/A-L 42,5 R & $5.00 \mathrm{~km}$ & Camion: $7.5 \tau, 02.38 \mathrm{MJ} / \mathrm{n}$ & $15.00 \mathrm{~km}$ & \\
\hline Cerámica & $2.00 \mathrm{~km}$ & \multirow{5}{*}{ Vagonetas (motor diesel) } & $300.00 \mathrm{~km}$ & \\
\hline Arena de río lavada & $2.00 \mathrm{~km}$ & & $15.00 \mathrm{~km}$ & \\
\hline Grava & $2.00 \mathrm{~km}$ & & $15.00 \mathrm{~km}$ & \\
\hline Refuerzo de acero corrugado B40oS & $2.00 \mathrm{~km}$ & & $30.00 \mathrm{~km}$ & \\
\hline Conectores de acero galvanizado & $2.00 \mathrm{~km}$ & & $30.00 \mathrm{~km}$ & \\
\hline Hormigón ligero HLE-25/P/20/IIa & $35.00 \mathrm{~km}$ & Camión: 7.5 t, $62.38 \mathrm{MJ} / \mathrm{h}$ & $8.00 \mathrm{~km}$ & Hormigonera: $10 \mathrm{~m}^{3}, 62.38 \mathrm{MJ} / \mathrm{h}$ \\
\hline Cerámica reciclada & $0.00 \mathrm{~km}$ & $\begin{array}{l}\text { Obtenido durante el proceso } \\
\text { de fabricación }\end{array}$ & $300.00 \mathrm{~km}$ & Camión: 7.5 t, $62.38 \mathrm{MJ} / \mathrm{h}$ \\
\hline
\end{tabular}

Igualmente, se ha dispuesto de la colaboración de diversos agentes sociales y económicos, como la Asociación para la Investigación y el Desarrollo Industrial de los Recursos aturales (AITEMIN) y la Asociación Española de fabricantes de Ladrillos y Tejas de Arcilla Cocida Hispalyt, además de diversas fábricas y empresas locales, como la Fábrica de Cerámica San Juan de Malpesa (Jaén) y la Fábrica Prebetong de Áridos y Hormigón de la Rinconada (Sevilla). Los agentes citados han facilitado información relevante para el proceso de cálculo, elaborándose un inventario con los datos.

Asimismo, los escenarios de transporte para las cuatro soluciones analizadas se muestran en la Tabla 4. En la etapa A4, las distancias consideradas se corresponden con la separa- ción entre la obra de rehabilitación y las fábricas existentes en el ámbito local (en el caso del cemento, hormigón, acero, áridos y grava, ubicadas en la provincia de Sevilla). En cuanto a la cerámica, la fábrica más cercana se encuentra en la provincia de Jaén, siendo el productor principal de cerámica de Andalucía. Por último, para la madera se ha considerado como punto de origen la comunidad autónoma de Galicia, primer productor de madera del país, ya que se constata que la madera "pinus pinaster" (pino gallego) se emplea con gran frecuencia en las edificaciones patrimoniales.

En la Figura 3 se puede observar la repercusión de las diferentes categorías de impacto analizadas por tonelada de cada material final empleado en las soluciones constructivas, con- 
siderando las etapas de la extracción de materias primas hasta el transporte a obra (A1 a A4). Esta Figura se ha elaborado con vistas a identificar las categorías de impacto que implican mayores cargas medioambientales, teniendo en cuenta los materiales utilizados y que, por tanto, deben ser analizadas con mayor detalle de cara al estudio de los forjados considerados.

Merece una especial atención el estudio de la ecotoxicidad del agua y del potencial de cambio climático (muy representativas en la cerámica, el cemento, el hormigón y la madera). Los materiales con mayores cargas medioambientales son el acero (corrugado y cincado), seguido del hormigón y del cemento. En los últimos puestos se sitúan la cerámica (conven- cional y reciclada) y la madera. En los aceros, es preocupante el impacto medioambiental ocasionado por radiaciones ionizantes.

A la vista de lo anterior, se concluye que las categorías de impacto más significativas que deben ser analizadas en las soluciones son: el potencial de cambio climático, la ecotoxicidad del agua, las radiaciones ionizantes sobre la salud humana, la acidificación y la eutroficiación del medio terrestre.

Los resultados del ACV por cada solución y etapa se muestran en las Figuras 4 a 7, indicando únicamente las categorías de impacto citadas con anterioridad. Estos resultados están ex-

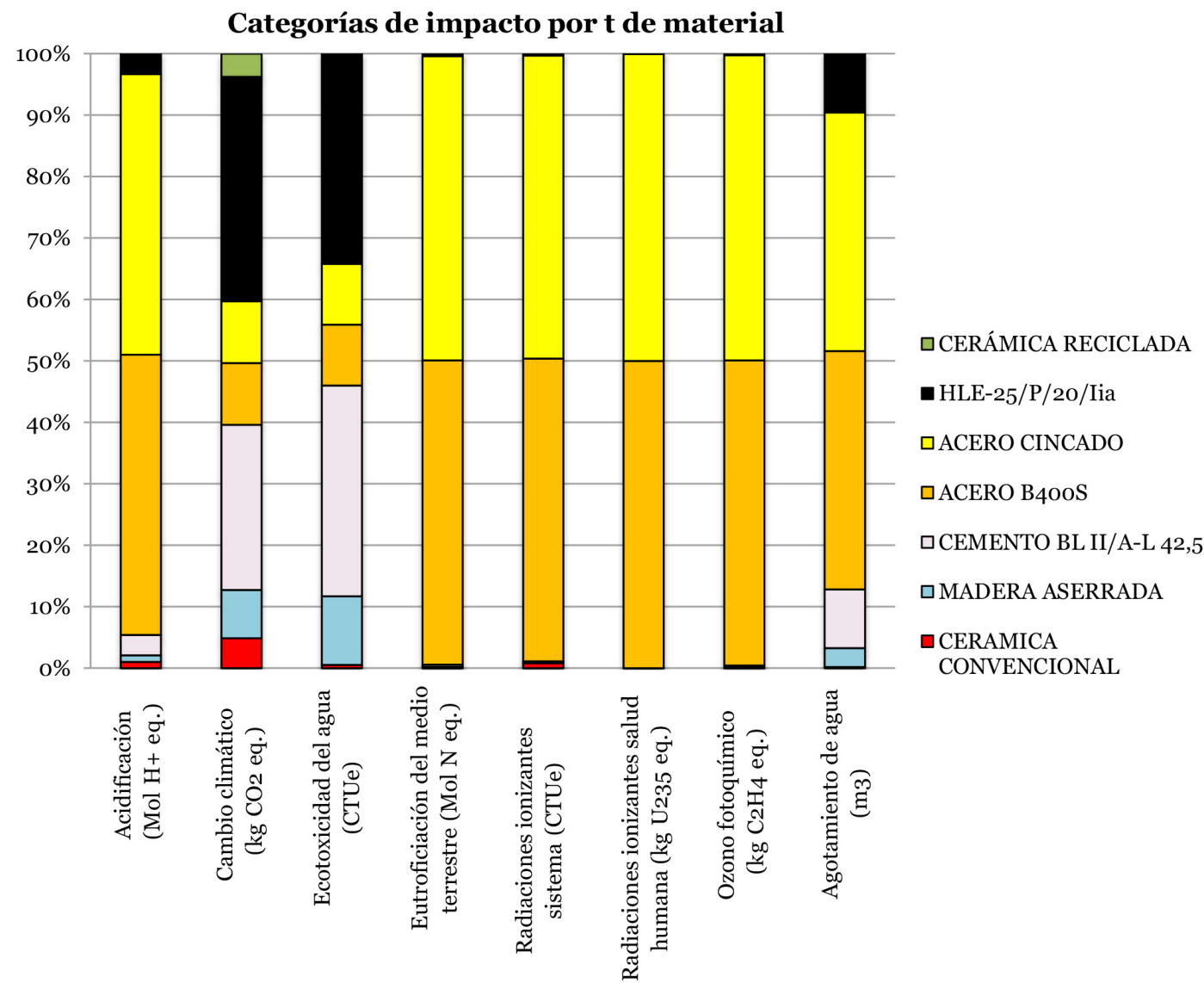

Figura 3. Repercusión de las diferentes categorías de impacto analizadas por cada tonelada de material que interviene en las soluciones de rehabilitación consideradas. Fuente: autores.

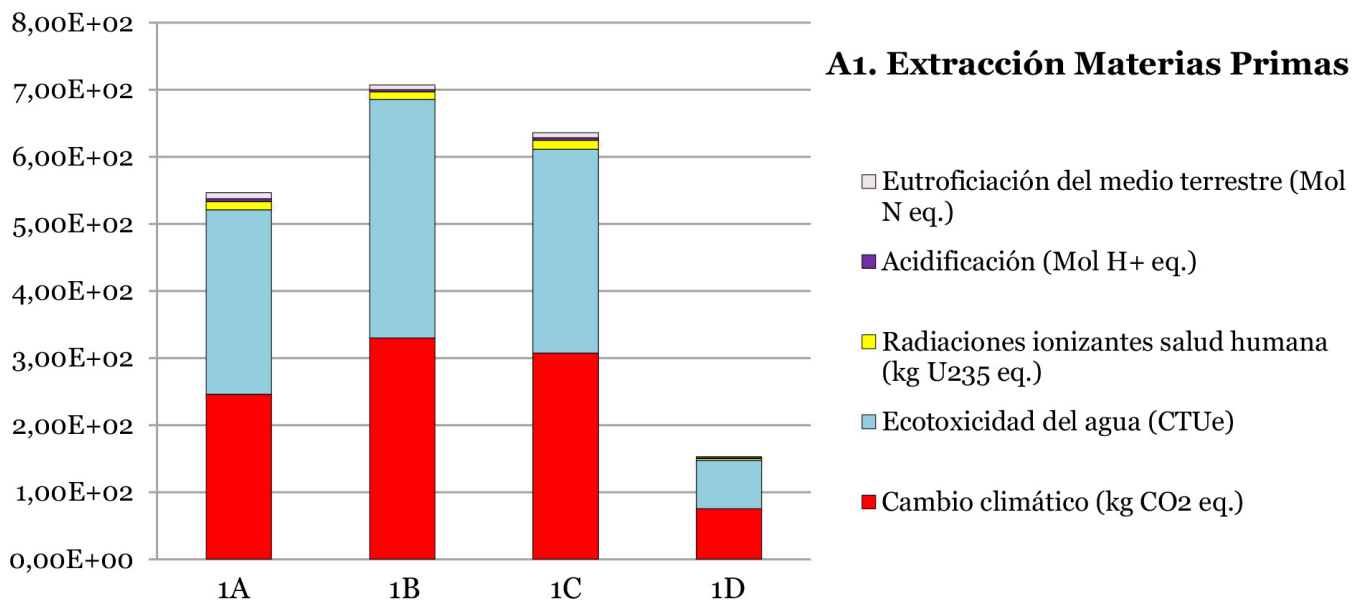

Figura 4. Principales impactos derivados de la extracción de materias primas. Fuente: autores. 


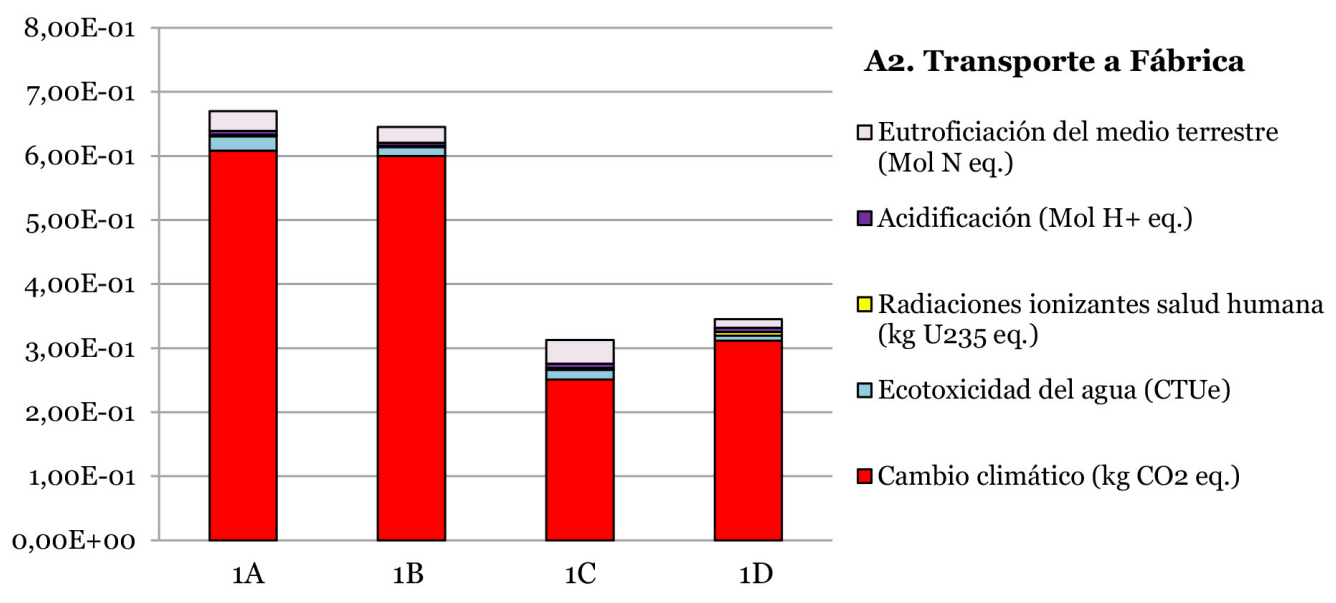

Figura 5. Principales impactos derivados del transporte de las materias primas a la planta de fabricación. Fuente: autores.

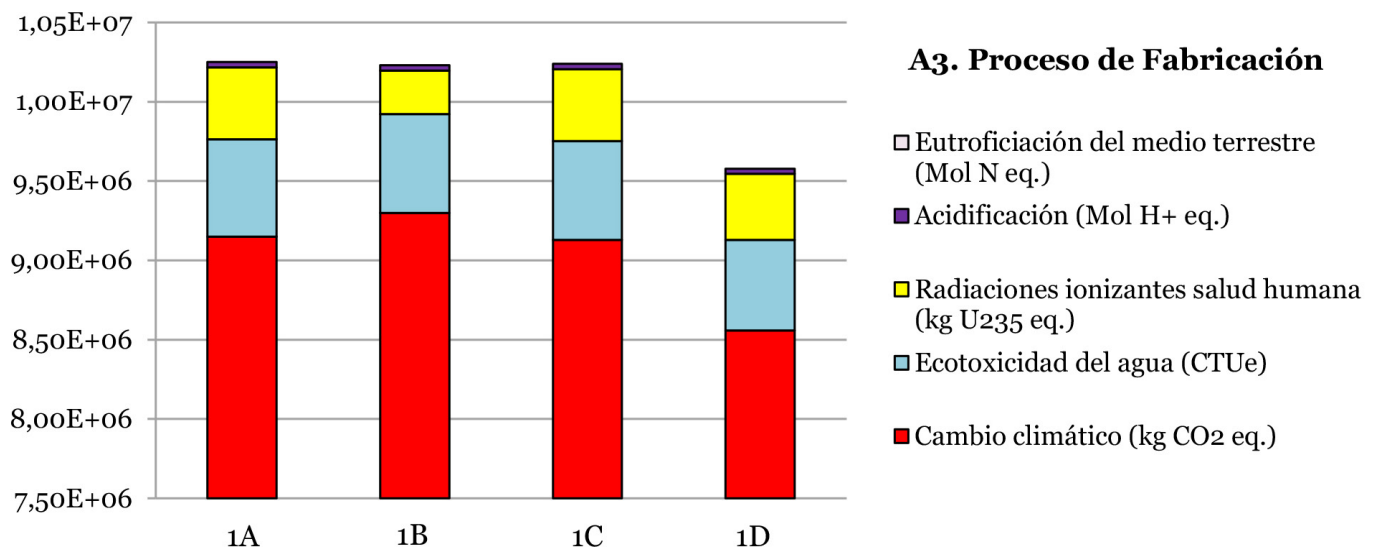

Figura 6. Principales impactos derivados del proceso de fabricación. Fuente: autores.

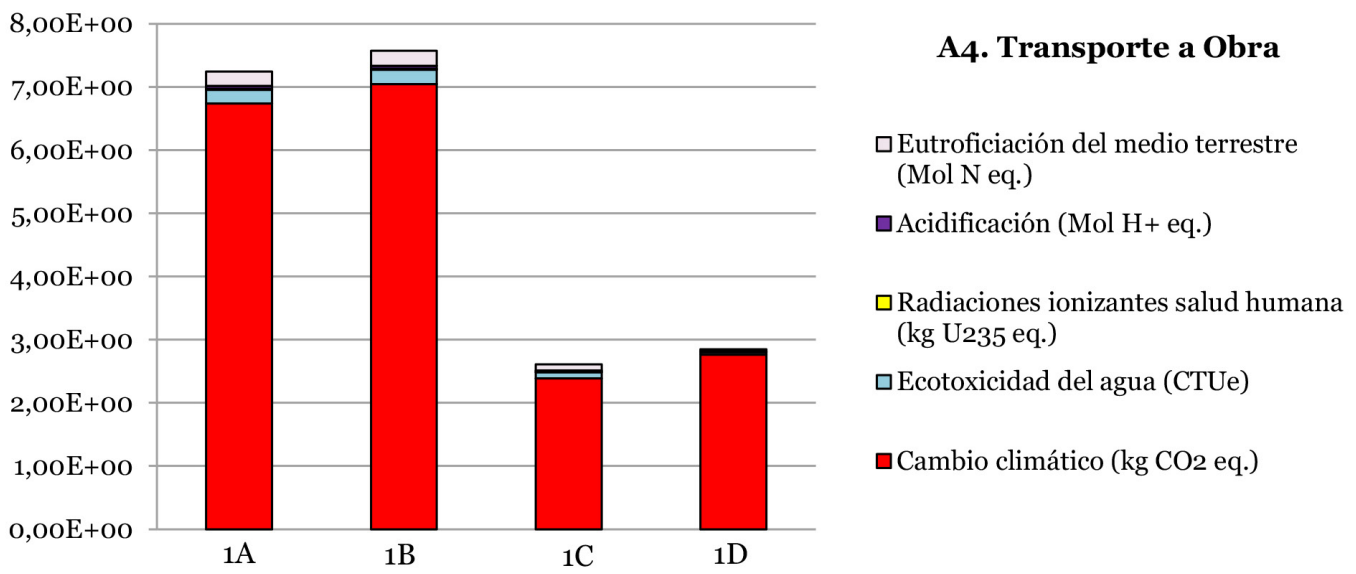

Figura 7. Principales impactos derivados del transporte a obra. Fuente: autores.

presados cuantitativa y gráficamente por $\mathrm{m}^{2}$ de forjado construido.

En las citadas figuras, puede observarse que la hipótesis $1 \mathrm{~b}$ (bovedilla cerámica maciza) representa claramente los valores más elevados, especialmente en cuanto a potencial de cambio climático y ecotoxicidad sobre el medio acuático, tanto en las etapas A1 y A4.

Es importante indicar que los valores en el resto de las etapas son igualmente elevados. Esta penalización se debe a que en dicha hipótesis, se ha dispuesto un mayor número de conectores de acero cincado por motivos estructurales, además, de un mayor espesor de la capa de compresión y del relleno de hormigón.

La hipótesis 1a (bovedilla cerámica aligerada), con niveles inferiores tanto de cerámica, al tratarse de un entrevigado convencional aligerado, como de hormigón, implica que las cargas medioambientales sean ligeramente inferiores en la etapa A1, y con valores muy similares a la solución $1 \mathrm{~b}$ (bovedilla cerámica maciza) en el resto de las etapas. 
La hipótesis 1c (bovedilla con losas cerámicas), presenta también impactos elevados en las etapas A1 y A3, debido a que el contenido de hormigón es incluso superior al de la hipótesis $1 \mathrm{~b}$ (bovedilla cerámica maciza).

En cambio, en las etapas restantes referidas al transporte (A2 y A4), se produce un descenso significativo de los impactos, ya que en esta hipótesis, al conservase la estructura horizontal de vigas de madera, no computa este material en el cálculo medioambiental (responsable de la penalización de las soluciones 1a y $1 \mathrm{~b}$ especialmente en la etapa $\mathrm{A} 4$, al considerarse en el cálculo distancias superiores a los $800 \mathrm{~km}$ ).

La opción 1d (bovedilla cerámica reciclada) es la que implica los menores impactos medioambientales en la etapa A1, puesto que la fase de extracción de materias primas y de producción ligadas a la cerámica se suprimen al reutilizarse residuos reciclados. Sin embargo, es importante indicar que se mantienen dichas etapas correspondientes al resto de materiales (hormigón, cemento, acero). Una particularidad de este caso es que para la fabricación de la bovedilla reciclada se ha incorporado cemento de forma adicional y con respecto a los niveles del resto de supuestos de rehabilitación, lo que hace que las cargas ambientales incrementen. Pese a ello, en la comparación global de todas las soluciones, siguen siendo cifras significativamente inferiores $y$, por tanto, mejores.

\section{CONCLUSIONES}

En el presente artículo, se ha llevado a cabo una valoración de los impactos medioambientales asociados a cuatro soluciones constructivas, empleadas en la rehabilitación de forjados con entrevigado abovedado característicos de las edificaciones de Sevilla de los S. XVII-XIX. Tres de las soluciones analizadas se basan en criterios de rehabilitación comúnmente empleados en la actualidad. En cambio, en la cuarta se dispone una pieza de entrevigado fabricada con un mortero de cerámica reciclada, siendo previamente analizada y ensayada.

Considerando un estudio del ciclo de vida de las etapas de A1 a A4 (desde la extracción de las materias primas hasta el transporte a obra, pasando por el proceso de fabricación), se concluye que en las cuatro soluciones las fases que más repercuten en el cálculo ambiental son el proceso de fabricación (A3), seguido de la etapa de obtención de materias primas (A1).

En el análisis pormenorizado de los materiales empleados en cada una de las hipótesis de rehabilitación, se ha de destacar los mayores impactos relativos al potencial de cambio climático durante la etapa de fabricación de la cerámica, además de la contribución a la ecotoxicidad del agua en el proceso productivo del cemento o el transporte a obra de la madera.

Es primordial resaltar los impactos debidos al hormigón, segundo material más contaminante del estudio y el de mayor presencia en las soluciones de rehabilitación, siendo las cate- gorías de impacto más características la ecotoxicidad del agua y el potencial de cambio climático. Igual importancia adquiere el cemento, material base empleado para la elaboración del mortero con residuos cerámicos reciclados, siendo responsable de la singular penalización medioambiental de la solución con entrevigado cerámico reciclado.

En contraposición, entre los materiales presentes en las soluciones de forjados, las barras de refuerzo y los conectores de acero representan escasamente un $2 \%$ de la superficie por $\mathrm{m}^{2}$ del forjado en las cuatro soluciones. Por ello, pese a que el acero presente elevadas cargas medioambientales por tonelada de producto fabricado, su influencia analizando el conjunto total es poco significativa.

Considerando la totalidad de las etapas, se concluye que la hipótesis con mayores impactos medioambientales asociados es la opción $1 \mathrm{~b}$, con cifras muy elevadas de potencial de cambio climático y ecotoxicidad del agua en todas las etapas. Esto es debido a su mayor contenido de materiales con altas cargas ambientales, caso del hormigón, de la cerámica convencional y del acero, en contraste con el resto de soluciones.

Las menores cargas ambientales se manifiestan en la solución con la bovedilla cerámica reciclada (1d), todo ello pese a la penalización del cemento comentada previamente. Esto se debe a que, al implementar residuos cerámicos reciclados, se descartan las fases A1 y A2, reduciéndose muy significativamente el aporte de kg CO2 equivalentes y de ecotoxicidad al agua. Además, la energía y recursos empleados en la fase A3 disminuyen considerablemente y, por consiguiente, en la misma línea también lo hacen los impactos ambientales. Además, hay que añadirle el hecho favorable de la conservación de las vigas de madera, lo que implica que tampoco se consideren en el cálculo ninguna de sus etapas. Esto último también era posible con la solución 1c (bovedilla con losas cerámicas), no obstante, son mayores los beneficios mecánicos y medioambientales de la hipótesis 1d (bovedilla cerámica reciclada).

Por todo lo anterior, este trabajo ha demostrado la viabilidad medioambiental y las ventajas patrimoniales de la bovedilla cerámica reciclada (1d), convirtiéndose en una solución plausible y competitiva en el sector de la rehabilitación actual. Puesto que desde un punto de vista global, es la solución que en el conjunto presenta las cargas medioambientales más favorables por $\mathrm{m}^{2}$ construido de forjado $\mathrm{y}$, por tanto, la que está ligada a mayores criterios de sostenibilidad y ecoficiencia.

\section{AGRADECIMIENTOS}

Nuestro agradecimiento al Grupo de Investigación TEP-205 Análisis y Evaluación de Sistemas Constructivos y Estructurales en la Arquitectura, de la E.T.S. de Arquitectura de la Universidad de Sevilla, que ha permitido realizar las pruebas y ensayos necesarios sobre la bovedilla cerámica reciclada, patentada por el Grupo.

\section{REFERENCIAS}

(1) Cabeza, L.F., Rincón, L., Vilariño, V., Pérez, G., Castell, A. (2014). Life cycle assessment (LCA) and life cycle energy analysis (LCEA) of buildings and the building sector: A review. Renewable and Sustainable Energy Reviews, 29: 394416. https://doi.org/10.1016/J.RSER.2013.08.037

(2) European Commission. (2011). EU Energy and Transport in Figures Pocketbook. Brussels, Belgium: Publications Office of the European Union. 
(3) Persson, J., Grönkvist, S. (2015). Drivers for and barriers to low-energy buildings in Sweden. Journal of Cleaner Production, 109: 296-304. https://doi.org/10.1016/J.JCLEPRO.2014.09.094

(4) INE. Statistics National Institute (2013). Survey on the collection and treatment of urban waste and the generation of waste in service and construction sectors in 2011 (In Spanish).

(5) Zabalza Bribián, I., Valero Capilla, A., Aranda Usón, A. (2011). Life cycle assessment of building materials: Comparative analysis of energy and environmental impacts and evaluation of the eco-efficiency improvement potential. Building and Environment, 46(5): 1133-1140. https://doi.org/10.1016/j.buildenv.2010.12.002

(6) Directive 2008/98/EC of the European Parliament and of the Council of 19 November 2008 on waste; Directive 2010/31/ EU of the European Parliament and of the Council of 19 May 2010 on the energy performance of buildings and Directive 2012/27/EU of the European Parliament and of the Council of 25 October 2012 on energy efficiency.

(7) Pereira-de-Oliveira, L.A., Castro-Gomes, J.P., Santos, P.M.S. (2012). The potential pozzolanic activity of glass and redclay ceramic waste as cement mortars components. Construction and Building Materials, 31: 197-203. https://doi. org/10.1016/j.conbuildmat.2011.12.110

(8) Medina, C., Sánchez de Rojas, M.I., Thomas, C., Polanco, J.A., Frías, M. (2016). Durability of recycled concrete made with recycled ceramic sanitary ware aggregate. Inter-indicator relationships. Construction and Building Materials, 105: 480-486. https://doi.org/10.1016/j.conbuildmat.2015.12.176

(9) European Commission (2012). Eurostat. Annual report on recycling statistics in the European Union.

(10) Russell-Smith, S.V., Lepech, M.D., Fruchter, R., Meyer, Y.B. (2015). Sustainable target value design: integrating life cycle assessment and target value design to improve building energy and environmental performance. Journal of Cleaner Production, 88: 43-51. https://doi.org/10.1016/j.jclepro.2014.03.025

(11) AENOR-ISO (2006). UNE-ISO 14040, Gestión ambiental. Análisis del ciclo de vida. Principios y marco de referencia y UNE-ISO 14044, Gestión ambiental. Análisis del ciclo de vida. Requisitos y directrices. Asociación Española de Normalización (AENOR).

(12) Ingwersen, W.W., Stevenson, M.J. (2012). Can we compare the environmental performance of this product to that one? An update on the development of product category rules and future challenges toward alignment. Journal of Cleaner Production, 24: 102-108. https://doi.org/10.1016/j.jclepro.2011.10.040

(13) Benveniste, G., Gazulla, C., Fullana, P., Celades, I., Ros, T., Zaera, V., Godes, B. (2011). Life cycle assessment and product category rules for the construction sector. The floor and wall tiles sector case study. Informes de la Construcción, 63(522): 71-81. https://doi.org/10.3989/ic.10.034

(14) Almeida, M.I., Dias, A.C., Demertzi, M., Arroja, L. (2015). Contribution to the development of product category rules for ceramic bricks. Journal of Cleaner Production, 92: 206-215. https://doi.org/10.1016/j.jclepro.2014.12.073

(15) Ibáñez-Forés, V., Bovea, M.D., Simó, A. (2011). Life cycle assessment of ceramic tiles. Environmental and statistical analysis. The International Journal of Life Cycle Assessment, 16(9): 916. https://doi.org/10.1007/s11367-0110322-6

(16) Souza, D.M. de, Lafontaine, M., Charron-Doucet, F., Bengoa, X., Chappert, B., Duarte, F., Lima, L. (2015). Comparative Life Cycle Assessment of ceramic versus concrete roof tiles in the Brazilian context. Journal of Cleaner Production, 89: 165-173. https://doi.org/10.1016/j.jclepro.2014.11.029

(17) Quinteiro, O., Almeida, M., Dias, A.C., Araújo, A., Arroja, L. (2014). The carbon footprint of ceramic products. In Assessment of Carbon Footprint in Different Industrial Sectors, pp. 113-150. Springer Publications. https://doi. org/10.1007/978-981-4560-41-2_5

(18) Han, B., Wang, R., Yao, L., Liu, H., Wang, Z. (2015). Life cycle assessment of ceramic façade material and its comparative analysis with three other common façade materials. Journal of Cleaner Production, 99: 86-93. https://doi. org/10.1016/j.jclepro.2015.03.032

(19) Rincón, L., Coma, J., Pérez, G., Castell, A., Boer, D., Cabeza, L.F. (2014). Environmental performance of recycled rubber as drainage layer in extensive green roofs. A comparative Life Cycle Assessment. Building and Environment, 74: 22-30. https://doi.org/10.1016/j.buildenv.2014.01.001

(20) Mateus, R., Neiva, S., Bragança, L., Mendonça, P., Macieira, M. (2013). Sustainability assessment of an innovative lightweight building technology for partition walls - Comparison with conventional technologies. Building and Environment, 67: 147-159. https://doi.org/10.1016/j.buildenv.2013.05.012

(21) Pajchrowski, G., Noskowiak, A., Lewandowska, A., Strykowski, W. (2014). Materials composition or energy characteristic - What is more important in environmental life cycle of buildings? Building and Environment, 72: 15-27. https:// doi.org/10.1016/j.buildenv.2013.10.012

(22) Zea Escamilla, E., Habert, G. (2015). Global or local construction materials for post-disaster reconstruction? Sustainability assessment of twenty post-disaster shelter designs. Building and Environment, 92: 692-702. https://doi. org/10.1016/j.buildenv.2015.05.036

(23) Such, A.V., García-Martínez, A., Sánchez-Montañés, B. (2015). Rehabilitación de edificios: revisión de modelos y la evaluación del impacto ambiental a través de análisis de ciclo de vida. In Proceedings of the II International congress on sustainable construction and eco-efficient solutions, Seville 25-27 may 2015, pp. 161-172. Sevilla: Universidad de Sevilla, Departamento de Construcciones Arquitectónicas.

(24) Rubio de Hita, P., Gálvez, F.P., Conde, M.J., Liñán, C.R. (2017). Reuse of Ceramic Demolition Waste in the Reconstruction of Planked Timber Floor Slabs. The Open Construction and Building Technology Journal, 11(1): 124-135. https:// doi.org/10.2174/1874836801711010124

(25) Gálvez, F.P., de Hita, P.R., Martín, M.O., Conde, M.M., Liñán, C.R. (2013). Sustainable restoration of traditional building systems in the historical centre of Sevilla (Spain). Energy and Buildings, 62: 648-659. https://doi.org/10.1016/j. enbuild.2012.05.009 
(26) Buyle, M., Braet, J., Audenaert, A. (2013). Life cycle assessment in the construction sector: A review. Renewable and Sustainable Energy Reviews, 26: 379-388. https://doi.org/10.1016/j.rser.2013.05.001

(27) OpenLCA versión 1.6.1. Software profesional de código abierto (Open Source Initiative) con licencia MPL (Mozilla Public License) para el Análisis de Ciclo de Vida. GreenDelta (Berlín), proyecto Prosuite del 7a Programa Marco de la Unión Europea.

(28) Patente $\mathrm{N}^{0}$ 201601008: Procedimiento para la fabricación de morteros con residuos cerámicos y su aplicación en una pieza de entrevigado para forjados con vigas de madera. Desarrollado por el Grupo de Investigación TEP-205 Análisis y Evaluación de Sistemas Constructivos y Estructurales en la Arquitectura, de la Universidad de Sevilla. Recuperado de http://www.grupo.us.es/grupotep205/

(29) Fichas de protección y catálogo complementario del Conjunto Histórico; Catalogación y alturas del Conjunto Histórico; Protección Especial: Ámbitos y elementos con valores singulares. Plan General de Ordenación Urbanística (PGOU) de Sevilla. 15 de marzo de 2007.

(30) Código Técnico de la Edificación, C.T. (2006). Documento Básico de Seguridad Estructural Madera (CTE DB SE M); Documento Básico de Seguridad Estructural (CTE DB SE), Documento Básico de Seguridad en caso de Incendio (CTE DB SI). Spanish Standard, Madrid.

(31) MetaBase de productos de la construcción que incluye el Banco BEDEC de productos de la Construcción. Instituto de Tecnología de la Construcción de Cataluña. Recuperado de https://itec.es/nouBedec.e/

(32) Base de Información Ambiental de productos y sistemas de la construcción. Instituto de Tecnología de la Construcción de Cataluña. Recuperado de https://itec.es/metabase/productos-sostenibles/19/218/m/

(33) Base de datos de carácter ambiental para productos de la construcción OpenDAP, creada en el programa europeo InData y con el apoyo del Instituto Eduardo Torroja de Ciencias de la Construcción y la Agencia Estatal Consejo Superior de Investigaciones Científicas. Recuperado de http://www.opendap.es/ 